\title{
Evolution of Survival Impact of Molecular Target Agents in Patients with Advanced Hepatocellular Carcinoma
}

\author{
Kazufumi Kobayashia, b Sadahisa Ogasawara ${ }^{a, b}$ Aya Takahashic ${ }^{c}$ Yuya Seko ${ }^{c}$ \\ Hidemi Unozawa $^{a}$ Rui Sato $^{d}$ Shunji Watanabe $^{e}$ Michihisa Moriguchi $^{c}$ Naoki Morimoto $^{e}$ \\ Satoshi Tsuchiya $^{d}$ Kenji Iwai ${ }^{d}$ Masanori Inoue ${ }^{f}$ Keita Ogawa ${ }^{a}$ Takamasa Ishino $^{a}$ \\ Terunao Iwanaga $^{a}$ Takafumi Sakuma ${ }^{a}$ Naoto Fujita ${ }^{a}$ Hiroaki Kanzaki ${ }^{a}$ Keisuke Koroki ${ }^{a}$ \\ Masato Nakamura ${ }^{a}$ Naoya Kanogawa ${ }^{a}$ Soichiro Kiyono ${ }^{a}$ Takayuki Kondo $^{a}$ Tomoko Saito $^{a}$ \\ Ryo Nakagawa $^{a}$ Eiichiro Suzuki $^{a}$ Yoshihiko Ooka ${ }^{a}$ Shingo Nakamoto ${ }^{a}$ Akinobu Tawada ${ }^{a} \mathrm{~g}$ \\ Tetsuhiro Chiba ${ }^{a}$ Makoto Arai ${ }^{\mathrm{a}, \mathrm{g}}$ Tatsuo Kanda ${ }^{\mathrm{a}, \mathrm{h}}$ Hitoshi Maruyama ${ }^{\mathrm{a}, \mathrm{i}}$ Kengo Nagashima ${ }^{\mathrm{j}, \mathrm{k}}$ \\ Jun Kato ${ }^{a}$ Norio Isoda ${ }^{e}$ Takeshi Aramaki ${ }^{d}$ Yoshito Itoh ${ }^{c}$ Naoya Kato $^{a}$ \\ ${ }^{a}$ Department of Gastroenterology, Graduate School of Medicine, Chiba University, Chiba, Japan; ${ }^{\text {bTranslational }}$ \\ Research and Development Center, Chiba University Hospital, Chiba, Japan; 'Department of Gastroenterology \\ and Hepatology, Kyoto Prefectural University of Medicine, Kyoto, Japan; dDivision of Interventional Radiology, \\ Shizuoka Cancer Center, Shizuoka, Japan; ${ }^{e}$ Division of Gastroenterology, Department of Medicine, Jichi Medical \\ University, Tochigi, Japan; fNumazu City Hospital, Shizuoka, Japan; 9Department of Medical Oncology, Graduate \\ School of Medicine, Chiba University, Chiba, Japan; hDivision of Gastroenterology and Hepatology, Department of \\ Medicine, Nihon University School of Medicine, Tokyo, Japan; 'Department of Gastroenterology, Juntendo University \\ School of Medicine, Tokyo, Japan; 'ंResearch Center for Medical and Health Data Science, The Institute of Statistical \\ Mathematics, Tokyo, Japan; kBiostatistics Unit, Clinical and Translational Research Center, Keio University Hospital, \\ Tokyo, Japan
}

\section{Keywords}

Sequential therapy · Sorafenib · Regorafenib · Lenvatinib · Ramucirumab

\begin{abstract}
Background and Aims: The prognosis of patients with advanced hepatocellular carcinoma (HCC) is expected to improve as multiple molecular target agents (MTAs) are now available. However, the impact of the availability of sequential MTAs has not been fully verified yet. Approach and $\boldsymbol{R e}$ sults: We retrospectively collected the data on the whole clinical course of 877 patients who received any MTAs as
\end{abstract}

first-line systemic therapy for advanced HCC between June 2009 and March 2019. The study population was divided into 3 groups according to the date of first-line MTA administration (period 1: 2009-2012, $n=267$; period 2: 2013-2016, $n=$ 352; period 3: 2017-2019, $n=258$ ). Then, we compared the number of MTAs used, overall survival (OS), and MTA treatment duration among the 3 groups. Analysis was also performed separately for advanced-stage and nonadvanced-

Kazufumi Kobayashi, Sadahisa Ogasawara, Aya Takahashi, Yuya Seko, Hidemi Unozawa, Rui Sato, Shunji Watanabe, Michihisa Moriguchi, Naoki Morimoto contributed equally to this work. Sadahisa Ogasawara shared co-first authorship.
(C) 2021 The Author(s).

Published by S. Karger AG, Basel

This is an Open Access article licensed under the Creative Commons Attribution-NonCommercial-4.0 International License (CC BY-NC) (http://www.karger.com/Services/OpenAccessLicense), applicable to the online version of the article only. Usage and distribution for commercial purposes requires written permission.
Correspondence to:

Kazufumi Kobayashi, kobayashi-kazufumi@chiba-u.jp

Sadahisa Ogasawara, ogasawaras@chiba-u.jp 
stage HCC. The proportion of patients who received multiple MTAs was remarkably increased over time $(1.1 \%, 10.2 \%$, and $42.6 \%$ in periods 1,2 , and 3 , respectively, $p<0.001)$. The median OS times were prolonged to $10.4,11.3$, and 15.2 months in periods 1,2 , and 3 , respectively $(p=0.016)$. Similarly, the MTA treatment durations were extended $(2.7,3.2$, and 6.6 months in periods 1,2 , and 3 , respectively; $p<0.001$ ). We confirmed that the correlation between OS and MTA treatment duration was strengthened (period 1: 0.395 , period 2: 0.505 , and period $3: 0.667$ ). All these trends were pronounced in the patients with advanced-stage HCC but limited in the patients with nonadvanced-stage HCC. Conclusions: The availability of multiple MTAs had steadily improved the prognosis of patients with advanced HCC patients, particularly advancedstage HCC patients.

(C) 2021 The Author(s)

Published by S. Karger AG, Basel

\section{Introduction}

Hepatocellular carcinoma (HCC) is ranked as the sixth most common neoplasm and the third leading cause of cancer-related death, with 841,080 cases diagnosed and 781,631 deaths in 2018 [1-3]. Its incidence is expected to increase and remains one of the major medical problems for the foreseeable future. Despite the advocacy of screening against high-risk populations with viral hepatitis carriers or/and cirrhosis, many patients are still diagnosed after they have reached advanced HCC $[4,5]$.

Even in the early 2000s, standard systemic therapies for advanced HCC had not been established, and the development of its treatment had lagged far behind that of treatments for other malignancies. In the late 2000s, 2 randomized, double-blind, placebo-controlled trials showed the survival benefit of sorafenib, with hazard ratios of $<0.70$ in patients with advanced HCC $[6,7]$. On the basis of these trials, sorafenib become the first standard systemic therapy to receive approval for advanced HCC worldwide. Since the approval of sorafenib, several largescale global trials of novel compounds have been conducted concurrently; however, none of these trials demonstrated a statistical hypothesis in both first- and second-line settings in advanced HCC [8]. These negative trials have created an unfortunate situation in that only a single standard agent is available for a long time. Since the mid-2010s, the negative circumstances have rapidly improved with multiple phase III trials of molecular target agents (MTAs) successfully demonstrating efficacy [912]. So far, 5 MTAs have been approved for use in the standard of care of patients with advanced HCC, with len- vatinib showing noninferiority in terms of survival benefit as compared with sorafenib in the frontline setting, and with regorafenib, cabozantinib, and ramucirumab demonstrating superiority to placebo in the second-line setting after sorafenib.

As multiple MTAs have become available for clinical use worldwide, sequencing treatment with several drugs is increasingly becoming a mainstream treatment strategy for patients with advanced HCC. However, to date, the magnitude of the survival impact of the availability of multiple MTAs in real-world practice has not been clarified yet. The aim of this study was to examine the change in the impact of MTAs on the survival of patients with advanced HCC in clinical practice.

\section{Patients and Methods}

Patients, Treatments with MTAs, and Clinical Parameters

The present study included patients with advanced HCC who received any MTAs as a first-line systemic therapy in 3 institutions (Chiba University Hospital, University Hospital Kyoto Prefectural University of Medicine, and Shizuoka Cancer Center) in Japan between June 2009 (at the time of sorafenib approval in Japan) and March 2019. We retrospectively collected clinical data from the databases of the institutions during the whole clinical courses of all the patients after first-line MTAs were administered. The data were locked on March 31, 2020. This study was approved by the Chiba University Research Ethics Committee (No. 3553). Ethics Committees of the other participating institutions also approved the protocol of this study. We had access to information that could identify individual patients during or after data collection. Patient data were anonymized and deidentified before analysis.

Four MTAs (sorafenib, regorafenib, lenvatinib, and ramucirumab) were approved for advanced HCC in Japan during the observation period of this study. The approval dates of sorafenib, regorafenib, lenvatinib, and ramucirumab by the Pharmaceuticals and Medical Devices Agency, which is the Japanese regulatory authority, were May 20, 2009; June 26, 2017; March 23, 2018; and June 18, 2019, respectively. In Japanese clinical practice, sorafenib and lenvatinib were used as MTAs for first-line therapy in accordance with the guidelines $[13,14]$. In addition, sorafenib was allowed to be administrated as post-treatment after lenvatinib, and lenvatinib was also approved to be used after sorafenib under the Japanese universal health-care system. Regorafenib was approved for patients with advanced HCC who have a history of systemic therapy and recommended for use after sorafenib on the basis of the included criteria of the RESORCE trial [9]. The use of ramucirumab was allowed for patients with advanced HCC with confirmed history of systemic therapy and alpha-fetoprotein levels of $\geq 400 \mathrm{ng} / \mathrm{mL}$. Cabozantinib was administered only to patients who participated in the clinical trial in this study, although it had not been approved in Japan within the observation period. Generally, the standard starting doses of MTAs were complied with each clinical trial. On the basis of the physicians' decisions, a reduced starting dose was allowed for some patients in clinical practice. Dynamic contrast-enhanced computed tomography or magnetic res- 
onance imaging was performed at baseline and every 1-3 months after starting treatment for evaluation of tumor response. Each MTA was continued until the physician determined clear disease progression on radiological imaging or until the occurrence of adverse events that prevented treatment continuation. Each physician made decisions on which treatment to subsequently administer as post-treatment, including MTA, transarterial chemoembolization (TACE), hepatic arterial infusion chemotherapy (HAIC), radiotherapy, resection, and local ablation. If local tumor control appeared to contribute to prolonged patient prognosis at the time of MTA failure, we selected the best treatment option among resection, local ablation, TACE, HAIC, or radiation. We opted to convert to other MTAs if both the patient's liver function and Eastern Cooperative Oncology Group Performance Status were maintained to the extent that MTAs were tolerated. We recommended best supportive care if the patient had a poor liver function or low Eastern Cooperative Oncology Group Performance Status at the time of MTA failure. If the post-treatment decision after MTA was in doubt, the treatment was reviewed and decided at the multidisciplinary team meeting in each institution.

We retrieved medical records and acquired data on the following clinical parameters: baseline demographic data, date of radiological progression, and cause and date of discontinuation of the first- and later-line treatments with MTAs. Moreover, data on post-treatment after MTA administration and date of death or last follow-up were collected in the present study. Radiological assessments were evaluated using both the response evaluation criteria in solid tumors (RECIST) version 1.1 and modified RECIST (mRECIST) $[15,16]$.

\section{Study Design}

We subdivided the patients with advanced HCC who received at least one MTA as first-line systemic therapy into 3 groups according to the start date of MTA administration (period 1: 20092012, period 2: 2013-2016, and period 3: 2017-2019). Then the overall survival (OS), progression-free survival (PFS) according to both RECIST version 1.1 and mRECIST, treatment duration with MTA, and post-MTA treatment survival for each period were compared. OS was defined as the time from the date of first-line MTA administration until the date of any cause of death. The censoring date was defined as the date of the last follow-up. PFS was established as the time from the date of first-line MTA administration until the date of radiological progression or any cause of death. The censoring date was defined as the date of last radiological assessment without progression. The duration of MTA treatment was calculated as the sum of the duration of MTA treatment. If patients were still taking any MTAs, a censor was assumed at the date of the last follow-up. Post-MTA treatment survival was determined as the time from the date of discontinuation of MTA as final-line therapy until the date of any cause of death. The censoring date was defined as the date of the last follow-up as well as that of OS. In the present study, the definition of advanced-stage HCC was set up using tumor factor of the Barcelona Clinic Liver Cancer staging system. We defined advanced-stage HCC as the identification of either extrahepatic metastasis (EHM) or macrovascular invasion (MVI) at baseline radiological findings during first-line MTA treatment. Other patients without either MVI or EHM were classified as nonadvanced-stage HCC.
Statistical Analyses

The Pearson $\chi^{2}$ test or Fisher exact test was used to compare demographic and clinical characteristics as appropriate. The Kaplan-Meier estimator was used to estimate median times of OS, PFS, duration of MTA treatment, and post-MTA treatment survival, and its 95\% confidence intervals (CIs). The log-rank test was used to compare survival curves. Univariate and multivariate Cox proportional-hazard models were used to estimate the hazard ratios for risk factors predicting OS. The Kendall's $\tau$ between OS and other endpoints (i.e., PFS and treatment duration of MTA) was used to assess the association between OS and other endpoints, and surrogacy at the individual level. The value of $\tau$ belongs to the interval $[-1,1]$, assumes 0 value when OS and other endpoints are independent, and has the value 1.00 when OS and other endpoints have perfect correlation. The Frank copula model that accounts for censoring was used to estimate $\tau$ [17]. A $p$ value of $<0.05$ was considered statistically significant. All statistical analyses were performed using the SPSS version 25 statistical software (SPSS-IBM, Chicago, IL, USA) and R software (version 4.0.3; R Foundation for Statistical Computing, Vienna, Austria).

\section{Results}

\section{Baseline Characteristics of the Whole Study}

Population and Subgroups according to Each Period

Table 1 shows baseline characteristics of 877 patients with advanced HCC who received at least one MTA in 3 Japanese institutions. The median age was 71.5 years, and $79.6 \%$ of the patients were male. Most of the patients were classified as having Child-Pugh class A (771 patients, $87.9 \%)$. On the basis of the radiological assessment before starting frontline MTA, $283(32.3 \%)$ and 434 patients (49.5\%) were found to have MVI and EHM, respectively. In the present study, 589 patients (67.2\%) were categorized into the group with advanced-stage HCC. Among advanced-stage HCC patients, 33.8\% (199 of 589 patients) were classified as advanced-stage HCC at the time of initial diagnosis and $66.2 \%$ (390 of 589 patients) progressed to advanced-stage HCC from early- or intermediate-stage HCC. Differences in tumor characteristics, including both size and number of intrahepatic lesions, and appearance patterns of MVI, were observed in patients with advanced-stage HCC at the time of initial diagnosis or not (Table 2). The median observation period of the whole study population was 9.6 months (95\% CI, 8.710.5).

After dividing into 3 periods, the proportion of elderly patients increased significantly, and the proportion of hepatitis $\mathrm{C}$ virus-positive patients decreased significantly over time. No significant differences in the frequency of Child-Pugh class A were found between the 3 periods. With the change of the time periods, the proportion of 
Table 1. Baseline characteristics of the study population

\begin{tabular}{|c|c|c|c|c|c|}
\hline & \multirow{2}{*}{$\begin{array}{l}\text { Whole study population } \\
N=877, n(\%)\end{array}$} & \multicolumn{3}{|c|}{ Period divided by start date of first-line MTA therapy, $n$ (\%) } & \multirow[t]{2}{*}{$p$ value } \\
\hline & & $\begin{array}{l}\text { period } 1(2009-2012) \\
n=267\end{array}$ & $\begin{array}{l}\text { period } 2(2013-2016) \\
n=352\end{array}$ & $\begin{array}{l}\text { period } 3(2017-2019) \\
n=258\end{array}$ & \\
\hline Age, $\geq 71.5$ years & $441(50.3)$ & $129(48.3)$ & $162(46.0)$ & $150(58.1)$ & 0.009 \\
\hline Sex, male & $698(79.6)$ & $213(79.8)$ & $293(83.2)$ & $192(74.4)$ & 0.028 \\
\hline HBV positive & $169(19.3)$ & 55 (20.6) & $68(19.3)$ & $46(17.8)$ & 0.723 \\
\hline ECOG-PS $\leq 1$ & $849(96.8)$ & $265(99.3)$ & $343(97.4)$ & $241(93.4)$ & 0.001 \\
\hline $\mathrm{MVI}$ & $283(32.3)$ & $95(35.6)$ & $114(32.4)$ & $74(28.7)$ & 0.239 \\
\hline EHM & $434(49.5)$ & $139(52.1)$ & $180(51.1)$ & $115(44.6)$ & 0.167 \\
\hline $\mathrm{AFP} \geq 400 \mathrm{ng} / \mathrm{mL}$ & $374(42.6)$ & $122(45.7)$ & $158(44.9)$ & $94(36.4)$ & 0.053 \\
\hline Prior treatment history & $753(85.9)$ & $235(88.0)$ & $308(87.5)$ & $210(81.4)$ & 0.041 \\
\hline
\end{tabular}

MTA, molecular target agent; HBV, hepatitis B virus; HCV, hepatitis C virus; ECOG-PS, Eastern Cooperative Oncology Group Performance Status; MVI, macrovascular invasion; EHM, extrahepatic metastasis; AFP, a-fetoprotein.

Table 2. Baseline characteristics of the patients with advanced-stage HCC by tumor stage at the time of initial diagnosis

\begin{tabular}{llrr}
\hline & \multicolumn{2}{l}{ Tumor stage at the time of initial diagnosis, $n(\%)$} & \multirow{2}{*}{$p$ value } \\
\cline { 2 - 3 } & advanced $(n=199)$ & not advanced $(n=390)$ & $<0.001$ \\
\hline Age, $\geq 71.5$ years & $73(36.7)$ & $204(52.3)$ & 0.259 \\
Sex, male & $164(82.4)$ & $306(78.5)$ & 0.005 \\
HBV positive & $58(29.1)$ & $74(19.0)$ & $<0.001$ \\
HCV positive & $49(24.6)$ & $176(45.1)$ & $<0.001$ \\
Child-Pugh class A & $163(81.9)$ & $354(90.8)$ & 0.141 \\
ECOG-PS $\leq 1$ & $189(95.0)$ & $378(96.9)$ & $<0.001$ \\
Tumor size $\geq 50$ mm & $117(58.8)$ & $94(24.1)$ & $<0.001$ \\
Tumors, $n \geq 8$ & $108(54.3)$ & $148(37.9)$ & $<0.001$ \\
MVI & $132(66.3)$ & $151(38.7)$ & 0.747 \\
EHM & $145(72.9)$ & $289(74.1)$ & $<0.001$ \\
AFP $\geq 400$ ng/mL & $112(56.3)$ & $154(39.5)$ & $<0.001$ \\
Prior treatment history & $108(54.3)$ & $379(97.2)$ & \\
Period divided by start date of first-line MTA & & & 0.878 \\
$\quad$ Period 1 & $64(32.2)$ & $123(31.5)$ & 0.566 \\
$\quad$ Period 2 & $85(42.7)$ & $157(40.3)$ & 0.427 \\
$\quad$ Period 3 & $50(25.1)$ & $110(28.2)$ & \\
\hline
\end{tabular}

HBV, Hepatitis B virus; HCV, hepatitis C virus; ECOG-PS, Eastern Cooperative Oncology Group Performance Status; MVI, macrovascular invasion; EHM, extrahepatic metastasis; AFP, a-fetoprotein; MTA, molecular target agent; HCC, hepatocellular carcinoma.

patients who received MTAs for advanced-stage HCC decreased (Fig. 1). Figure 2 shows the number of MTAs administered in each period. In terms of using multiple MTAs within the observation period, $10.2 \%$ and $42.6 \%$ of the patients received 2 or more MTAs in periods 2 and 3, respectively. By contrast, only $1.1 \%$ of the patients received multiple MTAs in period $1(p<0.001)$.

Sequential Therapy Prolonged the Survival of Advanced HCC Patients

\section{Transitions of OS by Period}

OS from the time of first-line MTA administration of the whole study population was 11.9 months $(95 \% \mathrm{CI}$, 10.9-12.9) in the present study. Figure 3 depicts the Kaplan-Meier curves of OS according to the 3 periods. The OSs in periods 1, 2, and 3 were 10.4 months $(95 \% \mathrm{CI}$, 8.2-12.5), 11.3 months (95\% CI, 9.8-12.8), and 15.2 
Fig. 1. Proportions of baseline tumor stages at the time of first-line MTA administration in each period. MTA, molecular target agent.
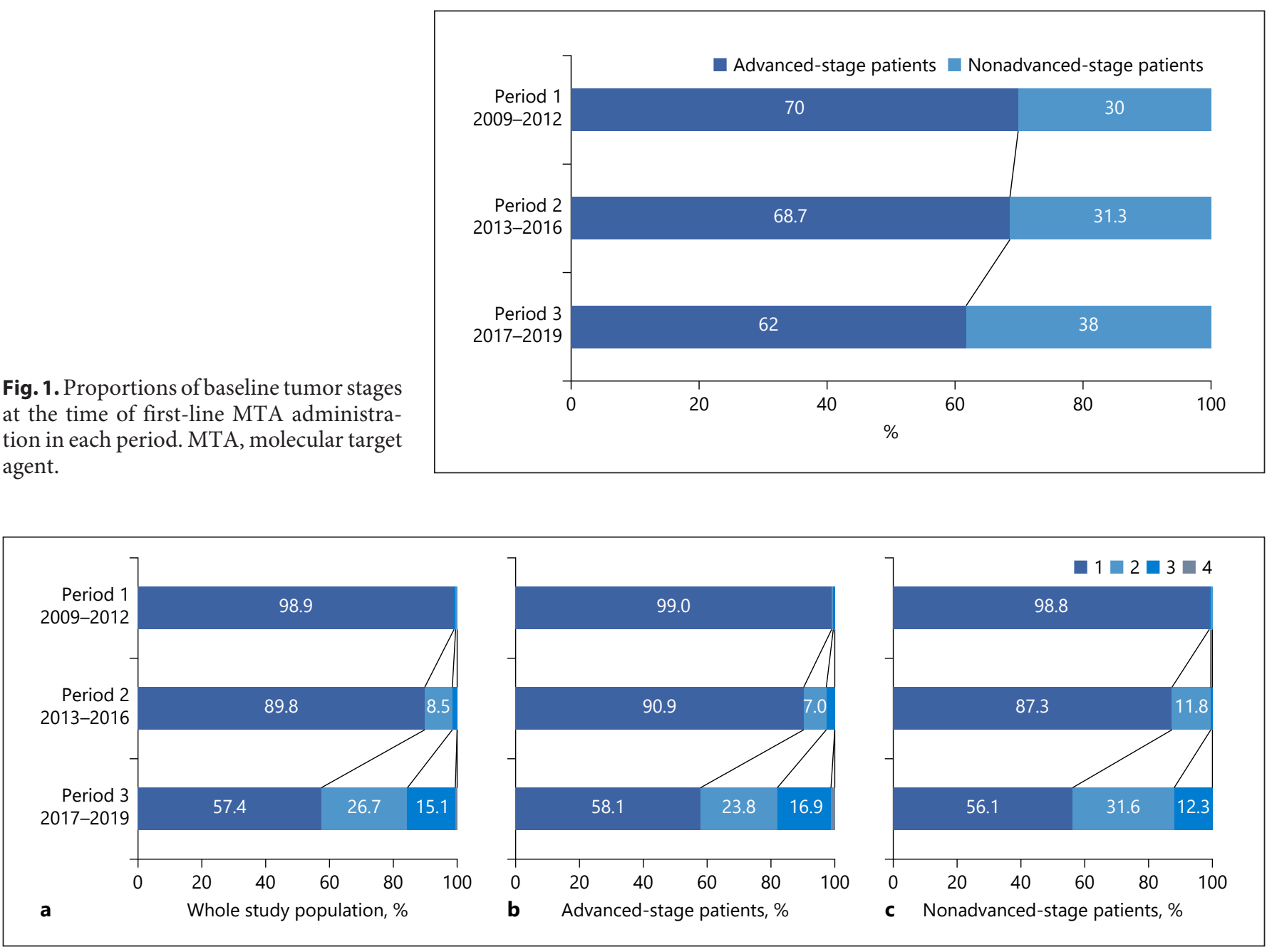

Fig. 2. Changes in the number of MTAs used in the clinical courses. Whole study population (a), patients with advanced-stage HCC (b), and patients with nonadvanced-stage HCC (c). HCC, hepatocellular carcinoma; MTA, molecular target agent.

months (95\% CI, 12.8-17.6), respectively (Fig. 3a). Significant differences in OS were found between the periods $(p=0.016)$. The OSs of the patients with advanced-stage HCC in periods 1, 2, and 3 were 8.7 months (95\% CI, 7.1-10.3), 10.7 months (95\% CI, 8.8-12.6), and 13.6 months (95\% CI, 10.4-16.8), respectively (Fig. 3b). Significant differences in OS were found among the periods ( $p=0.016)$. On the other hand, the OSs of the patients with nonadvanced-stage HCC were not significantly different between the 3 periods: period 1: 15.0 months [ $95 \%$ CI, 10.7-19.3], period 2: 13.1 months [95\% CI, 9.3-16.9], and period 3: 16.1 months [95\% CI, 12.1-20.1], $p=0.546$; Fig. 3c). The results of the Cox regression analyses for OS are shown in Table 3. In the present cohort, MTA administration in the latest period (period 3) was a significant factor of better prognosis. This result was also concordant with the result obtained from a cohort of 589 patients with advanced-stage HCC (online suppl. Table. 1; see www.karger.com/doi/10.1159/000519868 for all online suppl. material). On the other hand, in 288 patients without advanced-stage HCC, we could not observe an improvement of prognosis over time in the multivariate Cox regression analyses (online suppl. Table 2).

\section{Transitions of PFS after First-Line MTA, Duration of} MTA Treatment, and Post-MTA Treatment Survival by Period

Online supplementary Table 3 shows the PFS after frontline MTA treatment in accordance with both RECIST version 1.1 and mRECIST. The PFS of all the study 

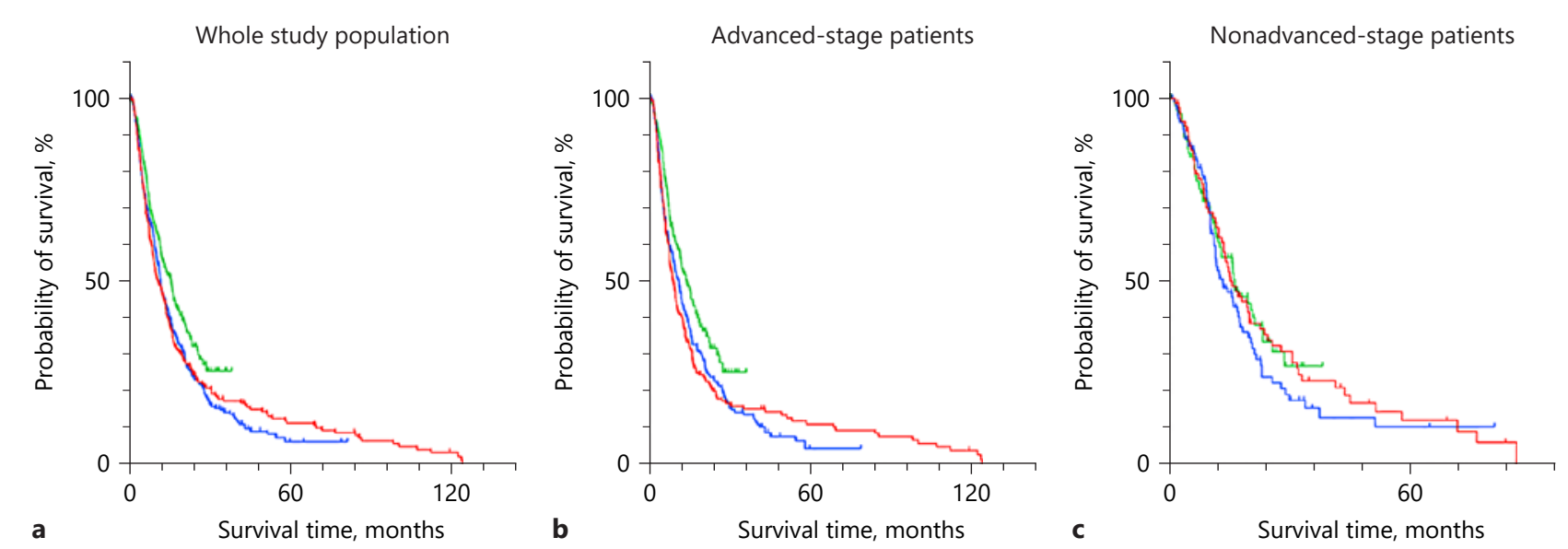

Fig. 3. Transitions of OS in each period. Whole study population (a), patients with advanced-stage HCC (b), and patients with nonadvanced-stage HCC (c). Red line: period 1, blue line: period 2, and green line: period 3. HCC, hepatocellular carcinoma; OS, overall survival.

Table 3. Cox proportional-hazard ratio for OS in patients with advanced HCC

\begin{tabular}{|c|c|c|c|}
\hline & Hazard ratio & $95 \% \mathrm{Cl}$ & $p$ value \\
\hline Age, $\geq 71.5$ years & 1.098 & $0.929-1.296$ & 0.273 \\
\hline Sex, male & 0.911 & $0.739-1.123$ & 0.383 \\
\hline HBV positive & 0.987 & $0.787-1.240$ & 0.913 \\
\hline HCV positive & 0.739 & $0.613-0.891$ & 0.002 \\
\hline \multicolumn{4}{|l|}{ Child-pugh score } \\
\hline 5 & Reference & & \\
\hline 6 & 1.634 & $1.362-1.960$ & $<0.001$ \\
\hline$\geq 7$ & 2.619 & $2.011-3.411$ & $<0.001$ \\
\hline $\mathrm{ECOG}-\mathrm{PS} \geq 2$ & 2.055 & $1.187-3.559$ & 0.010 \\
\hline \multicolumn{4}{|l|}{ Tumor status } \\
\hline Intrahepatic lesion only (no MVI and no EHM) & Reference & & \\
\hline EHM only (no intrahepatic lesion) & 0.956 & $0.730-1.300$ & 0.773 \\
\hline Intrahepatic lesion with MVI (no EHM) & 1.344 & $1.054-1.714$ & 0.017 \\
\hline Intrahepatic lesion with EHM (no MVI) & 1.145 & $0.919-1.426$ & 0.227 \\
\hline Intrahepatic lesion with both MVI and EHM & 1.949 & $1.504-2.527$ & $<0.001$ \\
\hline $\mathrm{AFP}, \geq 400 \mathrm{ng} / \mathrm{mL}$ & 1.382 & $1.165-1.640$ & $<0.001$ \\
\hline Prior treatment history & 1.025 & $0.799-1.315$ & 0.846 \\
\hline \multicolumn{4}{|l|}{ Period divided by start date of first-line MTA } \\
\hline Period 1 & Reference & & \\
\hline Period 2 & 1.005 & $0.833-1.213$ & 0.959 \\
\hline Period 3 & 0.763 & $0.611-0.952$ & 0.017 \\
\hline
\end{tabular}

Sequential Therapy Prolonged the Survival of Advanced HCC Patients 
Fig. 4. Evaluations of ALBI scores at 3 different time points (SOT with first-line MTA, EOT with first-line MTA, and EOT with any MTAs) during the clinical course of treatment with MTAs in the whole study population. ALBI, albumin-bilirubin; SOT, start of treatment; EOT, end of treatment; MTA, molecular target agent.

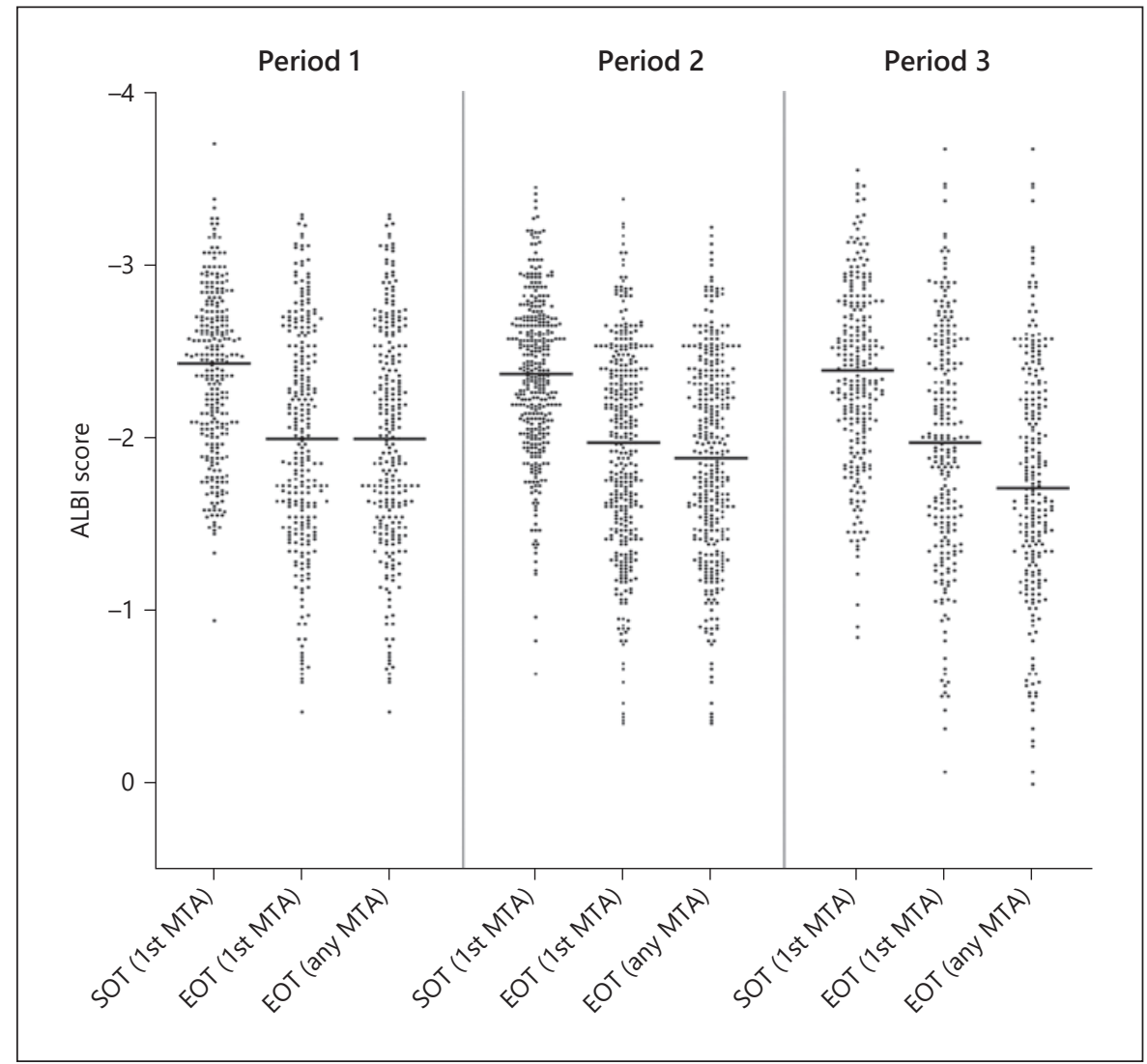

patients was 3.0 months (95\% CI, 2.7-3.3) and 3.0 months (95\% CI, 2.7-3.3), respectively. In all of the patients with any-, advanced-, nonadvanced-stage HCC, significant differences in PFS after first-line MTA treatment were found between the periods in both RECIST version 1.1 and mRECIST.

The duration of MTA treatment and post-MTA treatment survival in each period was evaluated in the present study (online suppl. Table 3). The duration of MTA treatment was prolonged significantly over time from period 1 through period 2 to period 3 . Corresponding to the results of the duration of MTA treatment, post-MTA treatment survival became shorter over time.

Changes in Liver Function at Baseline, the Time of First-Line MTA Discontinuation, and the End of All MTA Treatments

In the present study, we evaluated changes in liver function during the MTA treatments at 3 different time points (at baseline, the time of first-line MTA discontinuation, and the end of all MTA treatments) according to albumin-bilirubin (ALBI) score (Fig. 4). The median ALBI score at baseline and the time of first-line MTA dis- continuation had no significant difference between the 3 time periods $(-2.430,-2.370$, and -2.390 at baseline, $p=$ 0.957 and $-1.990,-1.970$, and -1.970 at first-line MTA discontinuation, $\mathrm{P} 0.458$ for periods 1,2 , and 3, respectively). On the other hand, the median ALBI score at the end of all MTA treatments significantly worsened over time $(-1.990,-1.880$, and -1.705 for periods 1,2 , and 3 , respectively, $p=0.001$ ). These results were also consistent with the stage-specific analyses of advanced- or nonadvanced-stage HCC (online suppl. Fig. 1).

\section{Post-MTA Treatment}

We also collected data regarding the post-MTA treatment in the present cohort (Table 4). In period 1, 48.7\% of the patients received alternative anticancer treatments which were defined as any treatment received after failure of standard systemic therapies, including clinical trials of systemic therapy, resection, local ablation, TACE, HAIC, and radiation therapy. The frequency of undergoing postMTA treatment had decreased through time (period 2: $34.8 \%$, period $3: 20.6 \%$ ). In the analyses of advanced-stage HCC and other HCC stages, the proportion of patients who received post-MTA treatment had decreased over 
Table 4. Transition of alternative anticancer therapy after MTA treatment

\begin{tabular}{llll}
\hline & \multicolumn{2}{l}{ Period divided by start date of first-line MTA, \% } \\
\cline { 2 - 3 } & period 1 & period 2 & period 3 \\
\hline Whole study population, any alternative anticancer treatment & 48.7 & 34.8 & 20.6 \\
TACE & 18.3 & 14.2 & 7.9 \\
HAIC & 4.2 & 10.6 & 8.5 \\
Systemic therapy including clinical trials & 23.2 & 8.2 & 3.2 \\
Others & 3.4 & 2.4 & 1.0 \\
Advanced-stage, any alternative anticancer treatment & 44.6 & 35.8 & 18.3 \\
TACE & 14.0 & 13.1 & 4.3 \\
HAIC & 2.2 & 10.9 & 8.7 \\
Systemic therapy including clinical trials & 24.7 & 9.6 & 4.3 \\
Others & 3.7 & 3.0 & 0.9 \\
Nonadvanced-stage, any alternative anticancer treatment & 58.4 & 32.7 & 24.3 \\
TACE & 28.6 & 16.8 & 13.5 \\
HAIC & 7.8 & 9.9 & 8.1 \\
Systemic therapy including clinical trials & 19.5 & 5.0 & 1.4 \\
Others & 2.6 & 1.0 & 1.4 \\
\hline
\end{tabular}

MTA, molecular target agent; TACE, transarterial chemoembolization; HAIC, hepatic arterial infusion chemotherapy.

time (advanced-stage, period 1: 44.6\%, period 2: 35.8\%, and period 3: $18.3 \%$; nonadvanced-stage, period 1:58.4\%, period 2: $32.7 \%$, and period $3: 24.3 \%$ ).

\section{Reliabilities between OS and PFS after First-Line \\ Therapy or the Duration of MTA Treatment}

Figure 5 indicates the reliabilities between the OS and PFS after first-line treatment or the duration of MTA treatment in the present cohort according to each period. In all the patients regardless of disease stage, the correlation between OS and the duration of MTA treatment increased over time (period 1: 0.395 , period 2: 0.505 , and period 3: 0.667 ), although the correlations between OS and PFS after first-line treatment calculated on the basis of both RECIST version 1.1 and mRECIST were approximately similar (RECIST version 1.1, period 1: 0.494 , period 2: 0.409 , and period 3: 0.522; mRECIST, period 1: 0.491, period 2: 0.410, and period 3: 0.534). These results were almost similar in the analyses by stage, which was divided into advancedstage HCC or other HCC stages (online suppl. Figs. 2, 3).

\section{Discussion}

The present study demonstrated that the OS of patients with advanced HCC had been prolonged as multiple MTAs were approved and used in clinical practice.
The trend of the improvement of the prognosis over time was more significant in advanced-stage HCC, defined as MVI or/and EHM recognized in the baseline radiological assessment during frontline MTA treatment. This population has had limited benefit from other alternative anticancer treatments. Therefore, most treatment guidelines worldwide recommend systemic therapies in this group of patients $[13,14,18-20]$. The fact that our results indicate a clear prognostic benefit of sequential MTA treatments in patients with advanced-stage HCC in realworld practice over time must be clinically meaningful.

Several reports have shown that sequential MTA therapies had a greater impact on the OS of patients with advanced HCC. As for lenvatinib and sorafenib in the frontline treatment for advanced HCC, the post hoc analyses of the REFLECT study demonstrated OS times for lenvatinib and sorafenib of 20.8 and 17.0 months, respectively, in patients who received subsequent anticancer medication after treatment with each agent, whereas patients who did not receive any subsequent medication after the first-line treatment had OS times of 11.5 and 9.1 months, respectively [21]. In the RESORCE trial, the median OS from starting sorafenib therapy was 26.0 months in patients who received regorafenib [9]. One study from Korea investigating clinical outcomes following treatment with regorafenib in patients with advanced HCC reported a 1 -year survival rate of $54.6 \%$ and a 2 -year survival rate 


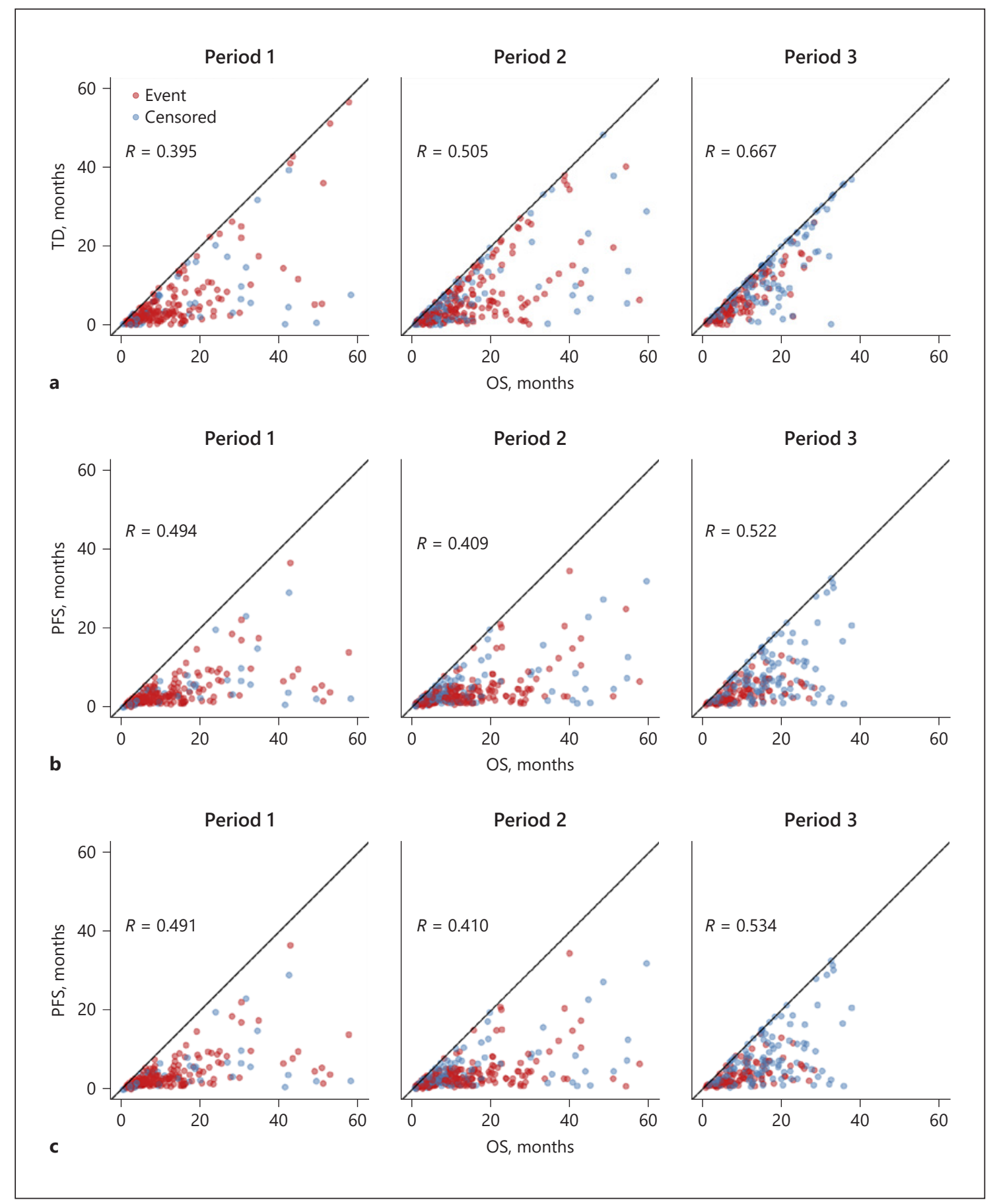

Fig. 5. Correlations between OS and duration of treatment with MTAs, between OS and PFS according to RECIST version 1.1, and between OS and PFS according to mRECIST in the whole study population. OS and duration of treatment with MTAs (a), OS and PFS (RECIST version 1.1) (b), and OS and PFS (mRECIST) (c). OS, overall survival; PFS, progression-free survival; RECIST, response evaluation criteria in solid tumors; mRECIST, modified RECIST; TD, treatment duration of molecular target agents; MTA, molecular target agent. 
of 51.5\% (median OS not reached) [22]. We also showed comparable results demonstrating that the OS from the start of regorafenib therapy was 17.3 months, with $45.9 \%$ of the patients who discontinued regorafenib shifting to lenvatinib as post-treatment [23]. The latest publication by Koroki et al. [24] documented that the OS of patients who migrated to post-treatment was better than that of patients who were treated with best supportive care among patients with advanced HCC who received lenvatinib as first-line MTA therapy. To the best of our knowledge, almost all the articles to date have focused on patients with advanced HCC who received a particular MTA and have reported limited OS values for individual treatments from the time these agents were administrated. On the other hand, consecutive cases of advanced HCC patients who have received any agent as a frontline treatment should be analyzed, as in this study, in order to validate the impact of sequential therapy itself on the prognosis of patients with advanced HCC. A report which included 85 patients with advanced HCC from Europe reported a median OS of 35 months in patients with sequential therapy, with a median OS of only 9 months in patients with single systemic treatment [25]. The present study is the first to comprehensively analyze the prognostic impact of MTAs over time in clinical practice by examining a large number of patients who received treatment with any MTA for advanced HCC.

In the present study, we demonstrated changes in the correlation between OS and the duration of MTA treatment, the OS and PFS after first-line therapy, and the prolonged prognosis of patients who received MTAs over time. The latest article by Hiraoka et al. [26] demonstrated OS and the duration of MTA treatment were highly correlated in patients with advanced HCC. Noteworthy in this study was the first report to show the correlativity between OS and the duration of MTA treatment increased as time passed. Moreover, the association between OS and the duration of MTA treatment was stronger than that between the OS and PFS after the first-line therapy over time. According to the analysis of clinical trials in the first-line treatment setting in patients with advanced HCC, the correlations of the OS and PFS after first-line therapy were moderate as compared with those in other cancers (Pearson correlation $[R]$ of 0.84 ) $[8,27$, $28]$. That is, the impact of post-treatment with the frontline agent on OS is likely to be considerable in patients with advanced HCC. Several phase III trials of promising compounds in advanced HCC have been considered unsuccessful due to the effect of post-treatment [29-31]. In line with these results, our data confirmed that the impact

Sequential Therapy Prolonged the Survival of Advanced HCC Patients of the frontline treatment had not changed significantly over time. In period 1 of this study, $98.9 \%$ of the patients received only a single MTA and $48.7 \%$ migrated to posttreatment with alternative anticancer treatment after MTA. On the other hand, only $20.6 \%$ of the patients moved to post-treatment with alternative anticancer treatment after MTA in period 3, when sequential MTA treatment became the mainstream. The comparison of liver function at baseline and the end of any MTA treatment according to ALBI score between the 3 periods revealed that liver function deterioration was most significant in period 3, when multiple MTAs would have continued to bond as much as possible. Taken together, the establishment of sequential therapy due to the advent of multiple MTAs and the prolonged treatment duration with MTAs must have contributed to the prolonged outcome of patients with advanced HCC treated with MTAs.

One of the most interesting and discussable outcomes from the present study was that the prognosis of the patients with nonadvanced-stage HCC did not improve over time despite the results of an increasing number of MTAs administered, the prolongation of MTA treatment duration, and the decreasing rates of migration of postMTA treatment, which were similar between the patients with advanced- and nonadvanced-stage HCC. The statistical power of our prognostic analysis appears inadequate in the cohort of nonadvanced-stage HCC patients due to a comparatively small sample size. Moreover, the observation time in period 3 with nonadvanced-stage patients may have been too short to detect the occurrence of the event in survival analysis, since nonadvanced-stage patients had a better prognosis than advanced-stage patients. The impact of MTAs with second- or later-line treatments might not have been greater than that of alternative anticancer treatments after first-line MTA therapy. Compared with the post-MTA treatment survival of patients with advanced- and nonadvanced-stage HCC over time, post-MTA survival was more shortened in the patients with nonadvanced-stage HCC, while both showed trends toward shortening. In other words, the alternative anticancer treatments after MTA therapy, mostly locoregional therapies such as TACE, were likely to have contributed to the prognosis of patients with nonadvanced-stage HCC, even after the emergence of multiple MTAs. Most patients with nonadvanced-stage HCC in this study were the patient population generally defined as patients with intermediate-stage HCC. According to guidelines used worldwide, the standard treatment for intermediate-stage HCC is TACE [13, 14, 18-20]. Disorderly repetition of TACE after recurrence of interme- 
diate-stage HCC not only is unlikely to be effective but also will worsen liver function $[32,33]$. Therefore, migration from TACE to systemic therapy at the appropriate timing has been recommended $[13,14,20]$. As all the participating institutions in the present study were centers with high volumes of HCC cases in Japan that perform comprehensive treatment by various specialists, we are also actively engaged in TACE. When selecting the MTA for intermediate-stage HCC (nonadvanced-stage in this study), the decision was based on the fact that TACE was not expected to be effective. The clinical impacts of the fact that sequential MTA treatments did not improve the prognosis would be meaningful in such patients. Nowadays, the atezolizumab plus bevacizumab regimen, which is a combination of anti-programmed cell death-ligand 1 antibody and anti-vascular epithelial growth factor antibody, has replaced the standard frontline MTA treatment in patients with advanced HCC [34]. The mainstream systemic therapy for HCC will become a combination immunotherapy with the advent of other regimens of combination immunotherapies in further clinical trials. We believe that combination immunotherapies, including atezolizumab combined with bevacizumab, can improve the prognosis of patients with intermediate-stage HCC who have indications for systemic therapies. By contrast, several clinical trials have been conducted to compare TACE plus combination immunotherapies with TACE in patients with intermediate-stage HCC $[35,36]$. TACE plus systemic therapy has been proposed as a promising treatment strategy for intermediate-stage HCC for a long time. However, the combination of TACE and MTA has not shown significant results in previous clinical trials [37-39]. We also expect that the TACE plus combination immunotherapies will dramatically change the treatment structure for intermediate-stage HCC.

In conclusion, the development of several MTAs and the use of multiple MTAs in the clinical course, so-called sequential therapy, markedly improved prognosis with prolongation of the duration of MTA treatment for advanced-stage HCC. The prognosis of these patients is expected to be further prolonged with the development of combination immunotherapies. By contrast, the impact of the prognostic improvement in patients with nonadvanced-stage HCC, mostly intermediate-stage HCC, was not as remarkable as expected. In this patient group, the development of innovative treatment strategies such as TACE plus combination immunotherapies is strongly required in addition to the prolongation of prognosis due to combination immunotherapies, represented by atezolizumab combined with bevacizumab.

\section{Acknowledgments}

The authors are grateful to the following people for their contributions to the data management: Satomi Nakamura, Yuka Iwase, and Ryoko Arai.

\section{Statement of Ethics}

All procedures performed in this study involving human participants were in accordance with the ethical standards of the institutional and/or national research committee and with the 1964 Helsinki declaration and its later amendments or comparable ethical standards. Formal consent by written signature was not required for this type of study based on Japanese regulation. The Chiba University Research Ethics Committee also approved this point. The Chiba University Research Ethics Committee approved this study (No. 3553).

\section{Conflict of Interest Statement}

Sadahisa Ogasawara received honoraria from Bayer, Leverkusen, Germany; Eisai, Tokyo, Japan; Eli Lilly, Indianapolis, IN, USA; Chugai Pharma, Tokyo, Japan; AstraZeneca, Cambridge, UK; and Merck \& Co., Inc., Kenilworth, NJ, USA, consulting or advisory fees from Bayer, Eisai, Merck \& Co., Inc., Chugai Pharma, Eli Lilly, and AstraZeneca, and research grants from Bayer, AstraZeneca, and Eisai. Michihisa Moriguchi received honoraria from Eisai, Bayer, Eli Lilly, and Chugai Pharma, and consulting or advisory fees from Eisai, Bayer, Eli Lilly, and Chugai Pharma. Naoki Morimoto received research grants from Eisai and Abbvie, Lake Bluff, IL, USA. Yoshihiko Ooka received honoraria from Eisai. Tatsuo Kanda received research grants from Towa Pharmaceutical, Osaka, Japan; Abbvie, Chugai Pharma, Daiichi Sankyo, Tokyo, Japan; and Shionogi, Osaka, Japan. Kengo Nagashima received a lecture fee from Pfizer, New York, NY, USA. Norio Isoda received research grants from Eisai and Abbvie. Takeshi Aramaki received speaker's bureau from Eli Lilly, Chugai Pharma, Termo, Milano, Italy; Eisai, and Taiho Pharma, Tokyo, Japan. Yoshito Itoh received speaker's bureau from MSD, Kenilworth, NJ, USA, Eisai, and Chugai Pharma; and research grants from MSD, Eisai, Bayer, and Chugai Pharma. Naoya Kato received honoraria from Bayer, Eisai, Sumitomo Dainippon Pharma, Tokyo, Japan, and Merck \& Co., Inc.; consulting or advisory fees from Bayer and Eisai; and research grants from Bayer and Eisai. The other authors have no conflicts of interest to declare.

\section{Funding Sources}

The authors did not receive any financial support.

\section{Author Contributions}

Kazufumi Kobayashi, Sadahisa Ogasawara, Aya Takahashi, Yuya Seko, Hidemi Unozawa, Rui Sato, Shunji Watanabe, Michihisa Moriguchi, Naoki Morimoto, Satoshi Tsuchiya, Kenji Iwai, 
Masanori Inoue, Keita Ogawa, Takamasa Ishino, Terunao Iwanaga, Takafumi Sakuma, Naoto Fujita, Hiroaki Kanzaki, Keisuke Koroki, Masato Nakamura, Naoya Kanogawa, Soichiro Kiyono, Takayuki Kondo, Tomoko Saito, Ryo Nakagawa, Eiichiro Suzuki, Yoshihiko Ooka, Shingo Nakamoto, Akinobu Tawada, Tetsuhiro Chiba, Makoto Arai, Tatsuo Kanda, Hitoshi Maruyama, and Kengo Nagashima made substantial contribution to conception and design, acquisition of data, analysis, and interpretation of data. Kazufumi Kobayashi, Sadahisa Ogasawara, Michihisa Moriguchi, Naoki Morimoto, Jun Kato, Norio Isoda, Takeshi Aramaki, Yoshito Itoh, and Naoya Kato drafted the article or revised it critically for important intellectual content. All authors approved the final version of the manuscript to be published.

\section{Data Availability Statement}

All data generated or analyzed during this study are included in this article and its online supplementary material files. Further enquiries can be directed to the corresponding author.

\section{References}

1 Bray F, Ferlay J, Soerjomataram I, Siegel RL, Torre LA, Jemal A. Global cancer statistics 2018: GLOBOCAN estimates of incidence and mortality worldwide for 36 cancers in 185 countries. CA Cancer J Clin. 2018;68:394424.

2 Forner A, Reig M, Bruix J. Hepatocellular carcinoma. Lancet. 2018;391:1301-14.

3 Villanueva A. Hepatocellular carcinoma. N Engl J Med. 2019;380(15):1450-62.

4 Bruix J, Reig M, Sherman M. Evidence-based diagnosis, staging, and treatment of patients with hepatocellular carcinoma. Gastroenterology. 2016;150:835-53.

5 Llovet JM, Kelley RK, Villanueva A, Singal AG, Pikarsky E, Roayaie S, et al. Hepatocellular carcinoma. Nat Rev Dis Primers. 2021; 7(1):6.

6 Llovet JM, Ricci S, Mazzaferro V, Hilgard P, Gane E, Blanc JF, et al. Sorafenib in advanced hepatocellular carcinoma. $\mathrm{N}$ Engl J Med. 2008;359:378-90

7 Cheng AL, Kang YK, Chen Z, Tso CJ, Qin S, Kim JS, et al. Efficacy and safety of sorafenib in patients in the Asia-Pacific region with advanced hepatocellular carcinoma: a phase III randomized, double-blind, placebo-controlled trial. Lancet Oncol. 2009;10:25-34.

8 Llovet JM, Montal R, Villanueva A. Randomized trials and endpoints in advanced HCC: role of PFS as a surrogate of survival. J Hepatol. 2019;70:1262-77.

9 Bruix J, Qin S, Merle P, Granito A, Huang YH, Bodoky G, et al. Regorafenib for patients with hepatocellular carcinoma who progressed on sorafenib treatment (RESORCE): a randomized, double-blind, placebo-controlled, phase 3 trial. Lancet. 2017;389:56-66.

10 Kudo M, Finn RS, Qin S, Han KH, Ikeda K, Piscaglia $F$, et al. Lenvatinib versus sorafenib in first-line treatment of patients with unresectable hepatocellular carcinoma: a randomized phase 3 non-inferiority trial. Lancet. 2018;391:1163-73.

11 Abou-Alfa GK, Meyer T, Cheng AL, ElKhoueiry AB, Rimassa L, Ryoo BY, et al. Cabozantinib in patients with advanced and progressing hepatocellular carcinoma. N Engl J Med. 2018;379:54-63.
12 Zhu AX, Kang YK, Yen CJ, Finn RS, Galle PR, Llovet JM, et al. Ramucirumab after sorafenib in patients with advanced hepatocellular carcinoma and increased $\alpha$-fetoprotein concentrations (REACH-2): a randomized, doubleblind, placebo-controlled, phase 3 trial. Lancet Oncol. 2019;20:282-96.

13 Kokudo N, Takemura N, Hasegawa K, Takayama T, Kubo S, Shimada M, et al. Clinical practice guidelines for hepatocellular carcinoma: the Japan Society of Hepatology 2017 (4th JSH-HCC guidelines) 2019 update. Hepatol Res. 2019;49:1109-13.

14 Omata M, Cheng AL, Kokudo N, Kudo M, Lee JM, Jia J, et al. Asia-Pacific clinical practice guidelines on the management of hepatocellular carcinoma: a 2017 update. Hepatol Int. 2017;11:317-70.

15 Eisenhauer EA, Therasse P, Bogaerts J, Schwartz LH, Sargent D, Ford R, et al. New response evaluation criteria in solid tumours: revised RECIST guideline (version 1.1). Eur J Cancer. 2009; 45:228-47.

16 Lencioni R, Llovet JM. Modified RECIST (mRECIST) assessment for hepatocellular carcinoma. Semin Liver Dis. 2010;30:52-60.

17 Burzykowski T, Molenberghs G, Buyse M, Geys H, Renard D. Validation of surrogate end points in multiple randomized clinical trials with failure time end points. J R Stat Soc: Ser C (Appl Stat). 2001;50(4):405-22.

18 Heimbach JK, Kulik LM, Finn RS, Sirlin CB, Abecassis MM, Roberts LR, et al. AASLD guidelines for the treatment of hepatocellular carcinoma. Hepatology. 2018;67:358-80.

19 Llovet JM, Villanueva A, Marrero JA, Schwartz M, Meyer T, Galle PR, et al. Trial design and endpoints in hepatocellular carcinoma: AASLD consensus conference. Hepatology. 2021;73 Suppl 1:158-91.

20 Galle PR, Forner A, Llovet JM, Mazzaferro V, Piscaglia F, Raoul J-L, et al. EASL clinical practice guidelines: management of hepatocellular carcinoma. J Hepatol. 2018;69(1): $182-236$.
21 Alsina A, Kudo M, Vogel A, Cheng AL, Tak WY, Ryoo BY, et al. Effects of subsequent systemic anticancer medication following firstline Lenvatinib: a post hoc responder analysis from the phase 3 REFLECT study in unresectable hepatocellular carcinoma. Liver Cancer. 2020;9:93-104.

22 Yoo C, Park JW, Kim YJ, Kim DY, Yu SJ, Lim TS, et al. Multicenter retrospective analysis of the safety and efficacy of regorafenib after progression on sorafenib in Korean patients with hepatocellular carcinoma. Invest New Drugs. 2019;37:567-72.

23 Ogasawara S, Ooka Y, Itokawa N, Inoue M, Okabe S, Seki A, et al. Sequential therapy with sorafenib and regorafenib for advanced hepatocellular carcinoma: a multicenter retrospective study in Japan. Invest New Drugs. 2020; 38:172-80.

24 Koroki K, Kanogawa N, Maruta S, Ogasawara $\mathrm{S}$, Iino $\mathrm{Y}$, Obu $\mathrm{M}$, et al. Posttreatment after Lenvatinib in patients with advanced hepatocellular carcinoma. Liver Cancer. 2021;10: 473-84. in press.

25 Kirstein MM, Scheiner B, Marwede T, Wolf C, Voigtländer T, Semmler G, et al. Sequential systemic treatment in patients with hepatocellular carcinoma. Aliment Pharmacol Ther. 2020;52:205-12.

26 Hiraoka A, Kumada T, Atsukawa M, Hirooka M, Tsuji K, Ishikawa T, et al. Important clinical factors in sequential therapy including lenvatinib against unresectable hepatocellular carcinoma. Oncology. 2019;97(5):277-85.

27 Terashima T, Yamashita T, Toyama T, Arai K, Kawaguchi K, Kitamura K, et al. Surrogacy of time to progression for overall survival in advanced hepatocellular carcinoma treated with systemic therapy: a systemic review and meta-analysis of randomized controlled trials. Liver Cancer. 2019;8:130-9.

28 Llovet JM, Villanueva A, Marrero JA, Schwartz M, Meyer T, Galle PR, et al. Trial design and endpoints in hepatocellular carcinoma: AASLD consensus conference. Hepatology. 2021;73 Suppl 1:158-91. 
29 Johnson PJ, Qin S, Park JW, Poon RT, Raoul JL, Philip PA, et al. Brivanib versus sorafenib as first-line therapy in patients with unresectable advanced hepatocellular carcinoma: results from the randomized phase III BRISKFL study. J Clin Oncol. 2013;31:3517-24.

30 Cainap C, Qin S, Huang WT, Chung IJ, Pan $\mathrm{H}$, Cheng $\mathrm{Y}$, et al. Linifanib versus sorafenib in patients with advanced hepatocellular carcinoma: results of a randomized phase III trial. J Clin Oncol. 2015;33:172-9.

31 Zhu AX, Rosmorduc O, Evans TR, Ross PJ, Santoro A, Carrilho FJ, et al. SEARCH: a phase III, randomized, double-blind, placebo-controlled trial of sorafenib plus erlotinib in patients with advanced hepatocellular carcinoma. J Clin Oncol. 2015;33:559-66.

32 Ogasawara S, Chiba T, Ooka Y, Kanogawa N, Motoyama T, Suzuki E, et al. Efficacy of sorafenib in intermediate-stage hepatocellu- lar carcinoma patients refractory to transarterial chemoembolization. Oncology. 2014;87: $330-41$.

33 Arizumi T, Ueshima K, Minami T, Kono M, Chishina $\mathrm{H}$, Takita M, et al. Effectiveness of sorafenib in patients with transcatheter arterial chemoembolization (TACE) refractory and intermediate-stage hepatocellular carcinoma. Liver Cancer. 2015;4(4):253-62.

34 Finn RS, Qin S, Ikeda M, Galle PR, Ducreux M, Kim TY, et al. Atezolizumab plus bevacizumab in unresectable hepatocellular carcinoma. N Engl J Med. 2020;382:1894-905.

35 Greten TF, Mauda-Havakuk M, Heinrich B, Korangy F, Wood BJ. Combined locoregional-immunotherapy for liver cancer. J Hepatol. 2019;70:999-1007.

36 Kudo M. Combination cancer immunotherapy in hepatocellular carcinoma. Liver Cancer. 2018;7(1):20-7.
37 Kudo M, Han G, Finn RS, Poon RT, Blanc JF, Yan L, et al. Brivanib as adjuvant therapy to transarterial chemoembolization in patients with hepatocellular carcinoma: a randomized phase III trial. Hepatology. 2014;60:1697707.

38 Lencioni R, Llovet JM, Han G, Tak WY, Yang J, Guglielmi A, et al. Sorafenib or placebo plus TACE with doxorubicin-eluting beads for intermediate stage HCC: the SPACE trial. J Hepatol. 2016;64:1090-8.

39 Kudo M, Cheng AL, Park JW, Park JH, Liang $\mathrm{PC}$, Hidaka $\mathrm{H}$, et al. Orantinib versus placebo combined with transcatheter arterial chemoembolisation in patients with unresectable hepatocellular carcinoma (ORIENTAL): a randomised, double-blind, placebo-controlled, multicentre, phase 3 study. Lancet Gastroenterol Hepatol. 2018;3:3746. 